Toshiaki Nakajima • Kikumi Iwaki • Tatsuhiko Kodama

Johji Inazawa $\cdot$ Mitsuru Emi

\title{
Genomic structure and chromosomal mapping of the human Site-1 protease (S1P) gene
}

\begin{abstract}
Site-1 protease (S1P) is a subtilisin-related enzyme that cleaves sterol regulatory element-binding proteins (SREBPs) in the lumen of endoplasmic reticulum, thereby initiating the release of transcriptionally active NH2-terminal fragments of SREBPs from membranes. In the experiments reported here, we localized the human $S 1 P$ gene to chromosome 16q24 by fluorescent in situ hybridization and radiation-hybrid mapping, and determined its genomic structure. This gene is more than $60 \mathrm{~kb}$ long and contains 23 exons and 22 introns. Its transcription-initiation site within exon 1 is separate from the initiation codon in exon 2. Analysis of the exon/intron structure revealed that the S1P gene consists of a mosaic of functional units: exon 1 encodes the $5^{\prime}$ non-translated region; exon 2 encodes the NH2-terminal signal sequence; and exons 2 and 3 encode the pro-peptide sequence that is released when $S 1 P$ is selfactivated by intramolecular cleavage. Exons 5-10 encode the subtilisin-homology domain that is critical for catalytic activity, and exon 23 encodes the transmembrane region. Analysis of the putative promoter region revealed a highly G/C-rich region containing a binding site for ADD1/ SREBP-1, as well as Sp1 and AP2 sites. Therefore, expression of the S1P gene may be under the control of SREBP1 , a key regulator of the expression of genes essential for intracellular lipid metabolism. Our data establish a basis for investigations to detect molecular variants in this gene that may alter levels of plasma lipoproteins and/or otherwise disrupt intracellular lipid metabolism.
\end{abstract}

T. Nakajima $\cdot$ K. Iwaki $\cdot$ M. Emi $(\bowtie)$

Department of Molecular Biology, Institute of Gerontology, Nippon Medical School, 1-396, Kosugi-cho, Nakahara-ku, Kawasaki 211-

8533, Japan

Tel. +81-44-733-5230; Fax +81-44-733-5192

e-mail: memi@nms.ac.jp.

T. Kodama

Department of Molecular Biology and Medicine, Research Center for Advanced Science and Technology, University of Tokyo, Tokyo, Japan

J. Inazawa

Medical Research Institute, Tokyo Medical and Dental University,

Yushima, Tokyo, Japan
Key words Site-1 protease (S1P) - Sterol regulatory element binding proteins (SREBPs)

\section{Introduction}

Cholesterol metabolism in mammalian cells is controlled by the sterol-regulated proteolysis of membrane-bound transcription factors called sterol regulatory element-binding proteins (SREBPs) (Sakai et al. 1996; Brown and Goldstein 1997; Sakai et al. 1998a,b). In this regulatory pathway, the first cleavage in SREBPs is made by site-1 protease (S1P), a membrane-bound subtilisin-related serine protease that cleaves SREBPs in a hydrophilic loop that projects into the lumen of the endoplasmic reticulum and other organelles. Cleavage by S1P separates the SREBP into two fragments, each of which has a single membrane-spanning sequence. This separation allows a second protease, designated site-2 protease (S2P), to cleave the NH2-terminal fragment at a position within its membrane-spanning sequence. The release of the NH2-terminal segment allows an SREBP to enter the nucleus, where it binds to enhancers and activates the transcription of genes encoding the low-density lipoprotein (LDL) receptor, as well as multiple enzymes of cholesterol and fatty acid biosynthesis (Yokoyama 1993; Guan et al. 1995; Osborne 1995; Ericsson et al. 1996; Kim and Spiegelman 1996; Vallett et al. 1996; Brown and Goldstein 1997). When sterols accumulate in cells, the cleavage of SREBPs by S1P is abolished; SREBPs remain attached to membranes, and the transcription of their target genes declines.

The site- 1 processing reaction is the target for the feedback-regulation of lipid biosynthesis and uptake in animal cells. This regulation is mediated by the sterol-sensing domain of SREBP cleavage-activating protein (SCAP) (Hua et al. 1996). This feedback regulation is disrupted in SRD-12B cells that harbor mutations in the S1P gene (Sakai et al. 1998a).

Given the central role of S1P in the regulation of lipid metabolism, molecular variants in the human $S 1 P$ gene 
would likely result in altered concentrations of plasma lipoproteins and/or in the derangement of lipid metabolism within tissues. As a first step in determining whether molecular variants in the SIP gene contribute to human disease, we determined the structure and chromosomal localization of the human S1P gene.

\section{Materials and methods}

Isolation of the human $\mathrm{S} 1 \mathrm{P}$ gene. We used a bacteriophage P1-derived artificial chromosome (PAC) library containing human genomic DNA pooled in a three-dimensional structure (Genome Systems, St. Louis, MO, USA), to isolate human S1P genomic clones. The three-dimensional PAC library was screened by a polymerase chain reaction (PCR) method described previously (Tsukamoto et al. 1998), using two oppositely oriented oligonucleotides (forward: $5^{\prime}-$ AAACCATCTTCATCGGTACTCCA-3' and reverse: 5'CTCGTTTAAAGGCTGTGGCTTG-3') that correspond, respectively, to nucleotides 3202-3224 and 3295-3316 of the human S1P cDNA (Genbank accession number, NM_003791). Escherichia coli containing positive clones were cultured in the presence of ampicillin, and PAC DNA was isolated as described elsewhere (Tsukamoto et al. 1998).

Sequencing of exon/intron junctions and the 5'-flanking region. Nucleotide sequences of exons and their bound- aries were determined by sequencing the PAC clone directly, using primers designed from the $S 1 P$ cDNA sequence. Sequencing was performed by the BigDye Terminator cycle sequencing method with a 377 ABI Prism automated DNA sequencer (Perkin Elmer, Norwalk, CT, USA). The size of each intron was determined by direct sequencing of the PAC genomic clone, or was estimated by PCR amplification of each intron, using LA Taq (Takara, Tokyo, Japan) and two oppositely oriented primers located in the exons immediately flanking each intron.

Fluorescence in situ hybridization (FISH). To assign the human S1P gene to its chromosomal location, a PAC clone carrying the human gene was used as the probe. Fluorescence in situ hybridization was carried out as described by Mukae et al. (1998). The PAC DNA was labeled with biotin16-deoxyuridine triphosphate (dUTP) by nick-translation and hybridized to denatured metaphase chromosomes at a final concentration of $25 \mathrm{ng} / \mathrm{ml}$ in $50 \%$ formamide, $10 \%$ dextran sulfate, $2 \times$ standard saline citrate (SSC), $0.2 \mathrm{mg} / \mathrm{ml}$ Cot-1 DNA (GIBCO/BRL, Frederick, MD, USA), $2 \mathrm{mg} / \mathrm{ml}$ salmon sperm DNA, and $2 \mathrm{mg} / \mathrm{ml}$ E. coli tRNA. Hybridized signals were detected with fluorescein isothiocyanate-avidin (Boehringer Mannheim, Indianapolis, IN, USA). Cells were counterstained with 4',6-diamidino-2-phenylindole, and the slides were examined through a Nikon epifluorescence microscope equipped with a charge-coupled device (CCD) camera (Photometrics, Tucson, AZ, USA). Images were captured with Quips (Vysis, Tokyo, Japan) software and processed with Adobe Photoshop 3.0 software.

Table 1. Exon/intron organization of the human S1P gene

\begin{tabular}{|c|c|c|c|c|}
\hline \multirow{2}{*}{$\begin{array}{l}\text { Exon } \\
\text { number }\end{array}$} & \multirow[b]{2}{*}{ Exon size (bp) } & \multicolumn{2}{|l|}{ Sequence at exon/intron junction ${ }^{\mathrm{a}}$} & \multirow[b]{2}{*}{ Intron size ${ }^{\mathrm{b}}(\mathrm{kb})$} \\
\hline & & 5' Splice donor & 3' Splice acceptor & \\
\hline 1 & $\sim 172$ & 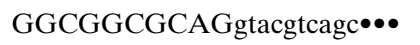 & $\bullet \bullet$ tgtttcatagATTGGCTCAC & 13.0 \\
\hline 2 & 487 & GTGGAATATGgtaatgatgt••• & 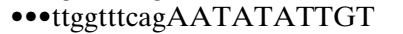 & 2.3 \\
\hline 3 & 258 & 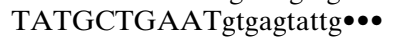 & $\bullet \bullet t$ tgcctgtagCTGACCCCAC & 3.0 \\
\hline 4 & 204 & GGATATACAGgtgtgtgcta $\bullet \bullet$ & $\bullet \bullet$ tccgaataagGTGCTAATGT & 1.7 \\
\hline 5 & 111 & CTGGACGATGgtgggtctca $\bullet \bullet$ & $\bullet \bullet$ tggactgcagGGTTGGGCCA & 0.4 \\
\hline 6 & 110 & CAATAATCAGgtaggtgttt••• & $\bullet \bullet a t t t a a c t a g G T A T C T T A C A$ & 1.3 \\
\hline 7 & 117 & TGTTGACAAGgtttgtggag ••• & $\bullet \bullet$ ccttttgtagGTGTGGGAAT & 2.8 \\
\hline 8 & 68 & CTCTTTATGGgtaagtacgc $\bullet \bullet$ & $\bullet \bullet c t c a a t a t a g C A C T C T G A A T ~$ & 0.7 \\
\hline 9 & 103 & 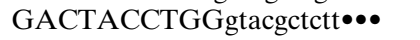 & $\bullet \bullet$ ttattcatagGAGCTACCAG & 2.0 \\
\hline 10 & 152 & TGTTAGTGAGgtgagtgctg••• & $\bullet \bullet$ tttcctccagCACAGTCCAG & 4.8 \\
\hline 11 & 162 & CACAGGCAAGgtcagtgcac••• & $\bullet \bullet t t t a t t t c a g T T T G A G C C C C$ & 10.0 \\
\hline 12 & 145 & TGTAGATAAGgtgaaacttc $\bullet \bullet$ & $\bullet \bullet t t t c c t g c a g C C T G A C T G G C$ & 2.8 \\
\hline 13 & 189 & AGAGACAGAGgtagggatat••• & $\bullet \bullet t t t t t t t t a g$ TCAAAAAATG & 1.1 \\
\hline 14 & 173 & CTTTAGACTGgtaagcacct••• & $\bullet \bullet$ tgtttttcagGAATGGTGAT & 1.4 \\
\hline 15 & 116 & AGTCAGTATGgtaagtggca••• & $\bullet \bullet$ tgttcatcagGCACTTTGCT & 0.6 \\
\hline 16 & 157 & AAAACACAAGgtactgttga $\bullet \bullet$ & $\bullet \bullet$ actttcccagGCAGTGGTGG & 1.2 \\
\hline 17 & 125 & AACCATGACAgtaaggctct $\bullet \bullet$ & $\bullet \bullet \bullet c t t t c c t a g T G T A T T A T G C$ & 0.7 \\
\hline 18 & 78 & AAGGACCAAGgtaaaggatg••• & $\bullet \bullet$ gttgtttcagGATTGGAGGT & 2.2 \\
\hline 19 & 141 & CGACAGAAGGgtaggaaaaa••• & $\bullet \bullet$ gacttcgcagACTGCTTTTG & 2.5 \\
\hline 20 & 132 & AGGATGGAAGgtgagtggat••• & $\bullet \bullet$ ctaaccacagGAAACCATCT & 1.2 \\
\hline 21 & 127 & CGGCGCCCAGgttagtgtcc •• & $\bullet \bullet \bullet t t c c t g a a g T A A C C T T T G G$ & 2.8 \\
\hline 22 & 131 & ATTCCTGGAGgtgagttctc $\bullet \bullet$ & $\bullet \bullet c c t c t t a c a g G G A T C A T G C C$ & 1.2 \\
\hline 23 & $\sim 880$ & & & \\
\hline
\end{tabular}

${ }^{\mathrm{a}}$ The sequences of exon/intron boundaries were determined as described in Materials and methods. Lowercase letters refer to intronic sequences, and uppercase letters to exonic sequences

${ }^{\mathrm{b}}$ Intron sizes were determined by direct sequencing of the P1-derived artificial chromosome (PAC) genomic clone, or were estimated by polynerase chain reaction (PCR) amplification, using LA Taq (TaKaRa, Tokyo, Japan) 

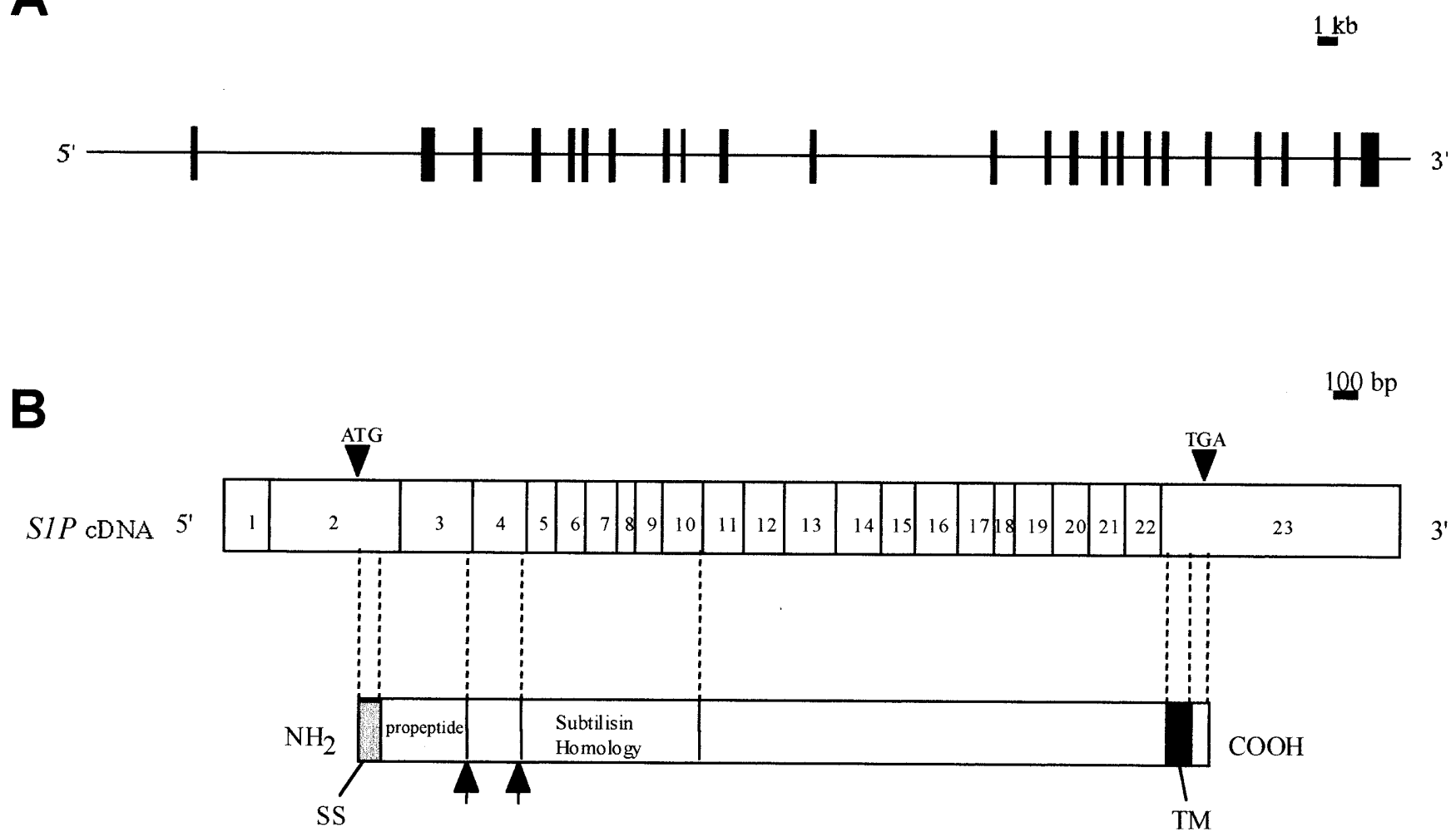

Fig. 1. A Genomic structure of the human $S 1 P$ gene. B Relationship between exon organization and functional domains of S1P. A translation initiation codon $(A T G)$ is present in exon 2, and a translation stop codon $(T G A)$ in exon 23. Upward arrows indicate $S 1 P$ processing sites. $S S$, Signal sequence; $T M$, transmembrane region

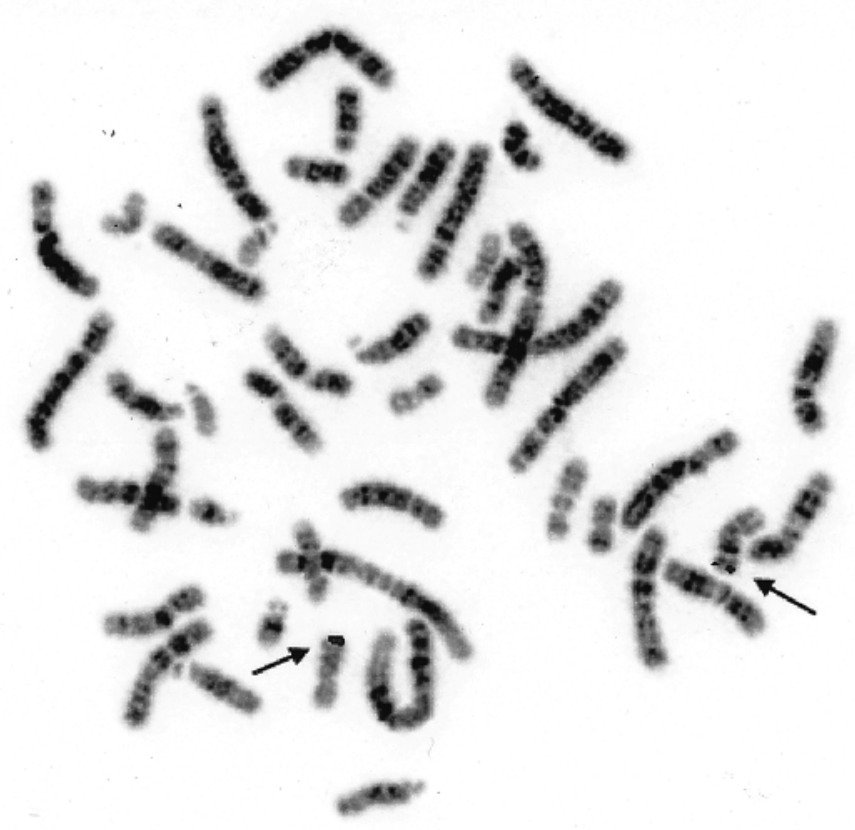

Fig. 2. Chromosomal localization of the human $S 1 P$ gene to $16 \mathrm{q}$ by fluorescence in situ hybridization on metaphase chromosomes
Radiation-hybrid mapping. The G3 RH mapping panel from the Stanford Human Genome Center (Boehnke et al. 1991), was used to confirm and define further the localization of the human $S 1 P$ gene. Typing was carried out using opposing primers located in the introns flanking exon 17 (forward: 5'-TGTTCCCTGTGTGTCTGTTACCAC-3'; reverse: $5^{\prime}$-GCTCTCACACCTAAGACCACGC-3'). The results of maximum-likelihood analysis were obtained by submitting the raw scores to http://www-shgc.stanford.edu.

\section{Results and discussion}

Cloning of the human S1P gene

More than $110 \mathrm{~kb}$ of the genomic region containing the entire human S1P gene was present in an isolated PAC clone. The gene is more than $60 \mathrm{~kb}$ long, about 15 times the size of its corresponding cDNA. It consists of 23 exons interrupted by 22 introns (Table 1 and Fig. 1A), exon 8 being the smallest, at $68 \mathrm{bp}$. Other exons ranged from 78 to around $880 \mathrm{bp}$. Sequences at the exon-intron boundaries for 


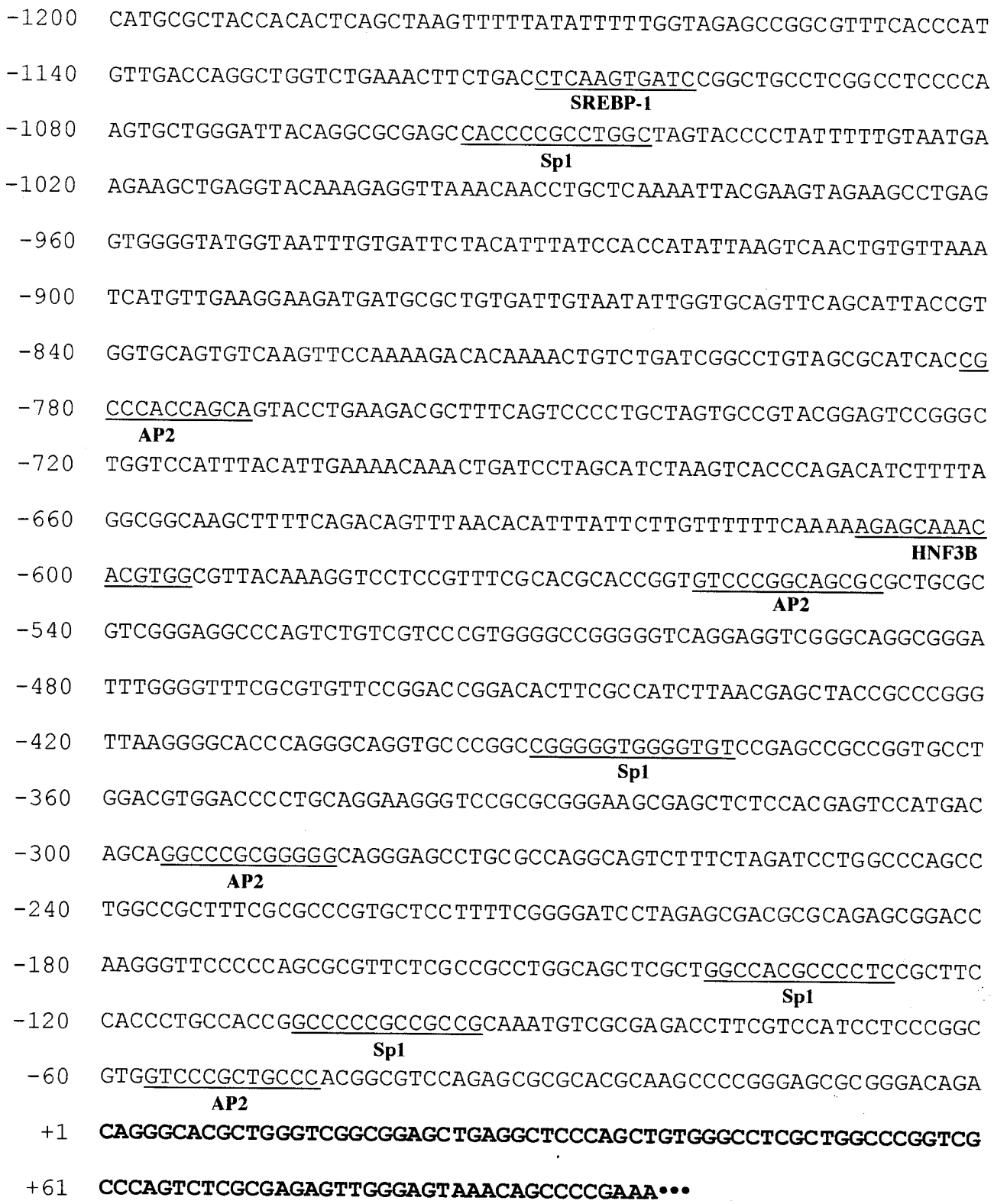

Fig. 3. Nucleotide sequence of the $5^{\prime}$ flanking region of S1P. Boldface letters indicate $5^{\prime}$ sequences of exon 1 . Nucleotide 1 is assigned to the putative transcription-initiation site. Putative binding motifs for Sp1,

all 22 introns are compatible with the consensus sequence for splicing junctions, including AG-GT (Mount 1982).

Comparison of the genomic sequence with the cDNA revealed that exon 1 and the $5^{\prime}$ half of exon 2 encode the 5 untranslated region (UTR); the protein-coding region extends from the $3^{\prime}$ portion of exon 2 through exon 22 , and exon 23 encodes both the carboxy-terminal coding region and the $3^{\prime}$ UTR (Fig. 1B). Exon 23 contains a polyadenylation site preceded by a polyadenylation signal.

Chromosomal mapping of the $S 1 P$ gene

A PAC genomic clone served as the probe to localize the $S 1 P$ gene by fluorescence in situ hybridization on
$\mathrm{AP} 2$, sterol regulatory element-binding protein (SREBP)-1, and HNF3B are indicated with underlines

metaphase chromosomes. Clear fluorescent signals were visualized on chromosomal band 16q24 (Fig. 2). The SIP gene was mapped more precisely by means of the G3 RH mapping panel of 83 hybrid cell lines from the Stanford Human Genome Center (Boehnke et al. 1991), through linkage to marker SHGC-30993 with a logarithm of differences (lod) score of more than 14.83). These results support data reported by Nagase et al. (1995).

Exon organization and protein domains

The introns of S1P interrupt the protein-coding sequence in such a way that many domains are encoded by distinct exons, as Fig. 1B indicates. Exon 1 encodes the $5^{\prime}$ non- 
translated region; part of exon 2 encodes the $22 \mathrm{NH} 2-$ terminal amino acids that constitute a signal sequence; the remainder of exon 2 and all of exon 3 encode the propeptide sequence that is synthesized as an inactive membrane-bound precursor and is self-activated by intramolecular cleavage to produce active enzyme (Cheng et al. 1999; Espenshade et al. 1999; Sakai et al. 1998a). Exons 5-10 encode the subtilisin-homology domain, whose aspartate 218, histidine 249 , and serine 414 residues are required for the catalytic activity of $S 1 P$ (Sakai et al. 1998a).

A region with no resemblance to other proteins is encoded by exons 11-22, and exon 23 encodes the transmembrane region and the $3^{\prime}$ UTR.

\section{Sequence of the $5^{\prime}$-flanking region of $S 1 P$}

The nucleotide sequence of the putative promoter of the S1P gene is given in Fig. 3. This sequence was examined with MatInspector V2.2 (Quandt et al. 1995) to identify any binding sites for transcription factors. This region is highly G/C-rich and contains Sp1 sites and AP2 sites, but no CAAT or TATA box characteristic of promoters of housekeeping genes (Dynan 1986). However, we identified an ADD1/SREBP-1 site (Yokoyama et al. 1993; Kim et al. 1995) in the 5'-flanking region of the $S 1 P$ gene. The SREBPs are a family of three closely related membranebound transcription factors that activate genes involved in the synthesis and uptake from plasma of cholesterol and unsaturated fatty acids, and regulate the transcription of genes encoding several enzymes in the cholesterol biosynthetic pathway (e.g., 3-hydroxy-3-methylgltarylcoenzyme A [HMG CoA] reductase, HMG CoA synthase, farnesyl diphosphate synthase, and squalene synthase; Guan et al. 1995; Osborne 1995; Ericsson et al. 1996; Vallett et al. 1996; Brown and Goldstein 1997). SREBPs also regulate the LDL receptor, which supplies cholesterol through re-ceptor-mediated endocytosis (Yokoyama 1993; Kim and Spiegelman 1996; Brown and Goldstein 1997). Transcriptional regulation by SREBP-1 therefore has a central role in lipid metabolism. For its part, S1Prelated proteolysis releases active fragments of SREBPs from membranes of the endoplasmic reticulum, and allows them to enter the nucleus (Hua et al. 1996). Although the transcriptional regulation of the S1P gene via SREBP-1 may be crucial for the regulation of lipid metabolism, its functional significance remains to be determined. The Sp1 sites present in its $5^{\prime}$-flanking region may act synergistically with SREBPs to activate expression of the LDL receptor gene (Sanchez et al. 1995; Yieh et al. 1995).

Our data establish a basis for investigations to discover molecular variants of the $S 1 P$ gene that may alter plasma lipoprotein levels and/or derange intracellular lipid metabolism.

Acknowledgments This work was supported by a Grant-in-Aid for Scientific Research in the Priority Area "Genome Science" from the Ministry of Education, Science, Sports, and Culture of Japan, by research grants for osteoporosis from the Ministry of Health and
Welfare of Japan, and by the Novartis Foundation for Gerontological Research.

\section{References}

Boehnke M, Lang K, Cox DR (1991) Statistical methods for multipoint radiation mapping. Am J Hum Genet 49:1174-1188

Brown MS, Goldstein JL (1997) The SREBP pathway: regulation of cholesterol metabolism by proteolysis of a membrane-bound transcription factor. Cell 89:331-340

Cheng D, Espenshade PJ, Slaughter CA, Jaen JC, Brown MS, Goldstein JL (1999) Secreted site-1 protease cleaves peptides corresponding to luminal loop of sterol regulatory element-binding proteins. J Biol Chem 274:22805-22812

Dynan WS (1986) Promoters for house-keeping genes. Trends Genet 2:196-197

Ericsson J, Jackson SM, Edwards PA (1996) Synergistic binding of sterol regulatory element-binding protein and NF-Y to the farnesyl diphosphate synthase promoter is critical for sterol-regulated expression of the gene. J Biol Chem 271:24359-24364

Espenshade PJ, Cheng D, Goldstein JL, Brown MS (1999) Autocatalytic processing of site-1 protease removes propeptide and permits cleavage of sterol regulatory element-binding proteins. J Biol Chem 274:22795-22804

Guan G, Jiang G, Koch RL, Shechter I (1995) Molecular cloning and functional analysis of the promoter of the human squalene synthase gene. J Biol Chem 270:21958-21965

Hua X, Nohturfft A, Goldstein JL, Brown MS (1996) Sterol resistance in $\mathrm{CHO}$ cells traced to point mutation in SREBP cleavage-activating protein. Cell 87:415-426

Kim JB, Spotts GD, Halvorsen YD, Shih HM, Ellenberger T, Towle HC, Spiegelman BM (1995) Dual DNA binding specificity of ADD1/SREBP1 controlled by a single amino acid in the basic helix-loop-helix domain. Mol Cell Biol 15:2582-2588

Kim JB, Spiegelman BM (1996) ADD1/SREBP1 promotes adipocyte differentiation and gene expression linked to fatty acid metabolism. Genes Dev 10:1096-1107

Mount SM (1982) A catalogue of splice junction sequences. Nucleic Acids Res 10:459-472

Mukae N, Enari M, Sakahira H, Fukuda Y, Inazawa J, Toh H, Nagata S (1998) Molecular cloning and characterization of human caspase-activated DNase. Proc Natl Acad Sci USA 95:9123-9128

Nagase T, Miyajima N, Tanaka A, Sazuka T, Seki N, Sato S, Tabata S, Ishikawa K, Kawarabayasi Y, Kotani H, et al (1995) Prediction of the coding sequences of unidentified human genes. III. The coding sequences of 40 new genes (KIAA0081-KIAA0120) deduced by analysis of cDNA clones from human cell line KG-1. DNA Res 2: $37-43$

Osborne TF (1995) Transcriptional control mechanisms in the regulation of cholesterol balance. Crit Rev Eukaryot Gene Expr 5:317-335

Quandt K, Frech K, Karas H, Wingender E, Werner T (1995) MatInd and MatInspector: new fast and versatile tools for detection of consensus matches in nucleotide sequence data. Nucleic Acids Res 23:4878-4884

Sakai J, Duncan EA, Rawson RB, Hua X, Brown MS, Goldstein JL (1996) Sterol-regulated release of SREBP-2 from cell membranes requires two sequential cleavages, one within a transmembrane segment. Cell 85:1037-1046

Sakai J, Rawson RB, Espenshade PJ, Cheng D, Seegmiller AC, Goldstein JL, Brown MS (1998a) Molecular identification of the sterol-regulated luminal protease that cleaves SREBPs and controls lipid composition of animal cells. Mol Cell 2:505-514

Sakai J, Nohturfft A, Goldstein JL, Brown MS (1998b) Cleavage of sterol regulatory element-binding proteins (SREBPs) at site-1 requires interaction with SREBP cleavage-activating protein. Evidence from in vivo competition studies. J Biol Chem 273:5785-5793

Sanchez HB, Yieh L, Osborne TF (1995) Cooperation by sterol regulatory element-binding protein and $\mathrm{Sp} 1$ in sterol regulation of low density lipoprotein receptor gene. J Biol Chem 270:1161-1169

Tsukamoto K, Ohta N, Shirai Y, Emi M (1998) A highly polymorphic CA repeat marker at the human interleukin 6 receptor (IL6R) locus. J Hum Genet 43:289-290 
Vallett SM, Sanchez HB, Rosenfeld JM, Osborne TF (1996) A direct role for sterol regulatory element binding protein in activation of 3 hydroxy-3-methylglutaryl coenzyme A reductase gene. J Biol Chem 271:12247-12253

Yieh L, Sanchez HB, Osborne TF (1995) Domains of transcription factor $\mathrm{Sp} 1$ required for synergistic activation with sterol regulatory element binding protein 1 of low density lipoprotein receptor promoter. Proc Natl Acad Sci USA 92:6102-6106

Yokoyama C, Wang X, Briggs MR, Admon A, Wu J, Hua X, Goldstein JL, Brown MS (1993) SREBP-1, a basic-helix-loop-helix-leucine zipper protein that controls transcription of the low density lipoprotein receptor gene. Cell 75:187-197 